\title{
Nilpotent variety of a reductive monoid
}

\author{
Mohan S. Putcha
}

Received: 5 March 2007 / Accepted: 15 June 2007 / Published online: 10 August 2007

(C) Springer Science+Business Media, LLC 2007

\begin{abstract}
In this paper we study the variety $M_{\text {nil }}$ of nilpotent elements of a reductive monoid $M$. In general this variety has a completely different structure than the variety $G_{u n i}$ of unipotent elements of the unit group $G$ of $M$. When $M$ has a unique non-trivial minimal or maximal $G \times G$-orbit, we find a precise description of the irreducible components of $M_{\text {nil }}$ via the combinatorics of the Renner monoid of $M$ and the Weyl group of $G$. In particular for a semisimple monoid $M$, we find necessary and sufficient conditions for the variety $M_{n i l}$ to be irreducible.
\end{abstract}

Keywords Reductive monoid · Nilpotent variety $\cdot$ Unipotent variety $\cdot$ Renner monoid · Weyl group

Mathematics Subject Classification (2000) Primary 20M10 · Secondary 20G99

\section{Introduction}

For a reductive group $G$, the variety $G_{u n i}$ of unipotent elements plays an important role, both in the study of structure of $G$ and in the representation theory of the associated finite reductive groups, cf. [2]. In particular $G_{\text {uni }}$ is an irreducible variety with finitely many conjugacy classes. We are interested in the variety $M_{n i l}$ of nilpotent elements of a reductive monoid $M$ with unit group $G$. Nilpotent elements are a natural object of study since an analogue of the Fitting decomposition is valid for reductive monoids, cf. [7, Theorem 4.1]. When $M$ is the multiplicative monoid of all $n \times n$ matrices, the two varieties $M_{n i l}$ and $G_{u n i}$ are isomorphic. But this is where the analogy ends. In general any connections between nilpotent and unipotent elements is either

M.S. Putcha $(\bowtie)$

Department of Mathematics, North Carolina State University, Box 8205, Raleigh, NC 27695-8205,

USA

e-mail: putcha@math.ncsu.edu 
non-existent or subtle. The variety $M_{\text {nil }}$ need not be irreducible and the number of conjugacy classes in $M_{\text {nil }}$ is generally infinite. There is of course also the variety $\mathfrak{g}_{\text {nil }}$ of nilpotent elements of the Lie algebra $\mathfrak{g}$ of $G$. However $\mathfrak{g}_{n i l}$ is much more closely related to $G_{u n i}$ than to $M_{\text {nil }}$.

We began the study of the variety $M_{\text {nil }}$ in [10], in particular finding a close connection with the 'conjugacy decomposition' of $M$. In this paper we obtain an explicit description of the irreducible components of $M_{\text {nil }}$ when $M$ is $\mathcal{J}$-irreducible (has a unique minimal non-zero $G \times G$-orbit) or $\mathcal{J}$-coirreducible (has a unique maximal $G \times G$-orbit not equal to $G$ ). In the first case each component of $M_{\text {nil }}$ intersects a unique maximal $\mathcal{J}$-class $(G \times G$-orbit) $J \neq G$. Moreover for a given $J$, at most three components of $M_{\text {nil }}$ intersect $J$, and we give a precise description of each. For $\mathcal{J}$-coirreducible monoids, the irreducible components of $M_{\text {nil }}$ are described in terms of the Coxeter elements of the Weyl group that are also minimal length coset representatives of an associated parabolic subgroup. In particular we find necessary and sufficient conditions for $M_{\text {nil }}$ to be irreducible when $M$ is semisimple (has a one dimensional center).

\section{Preliminaries}

Let $\kappa$ be an algebraically closed field. By an irreducible algebraic monoid, we mean a monoid $M$ with zero 0 , whose underlying set is an irreducible affine variety and the product map is a morphism. We will say that $M$ is a reductive monoid if the unit group $G$ of $M$ is reductive. Equivalently $M$ is regular $(a \in a M a$ for all $a \in M$ ). We refer to $[9,16,17]$ for the general theory of reductive monoids. For a reductive monoid $M$, the center $Z(G)$ of $G$ has dimension at least one. $M$ is said to be semisimple if the center $Z(G)$ of $G$ is one dimensional. Equivalently 0 and 1 are the only central idempotents of $M$. The multiplicative monoid $M_{n}(\kappa)$ of all $n \times n$ matrices over $\kappa$ is such an example. More generally if $G_{0} \subseteq G L_{n}(\kappa)$ is a semisimple group then the lined closure $\overline{\kappa G_{0}}$ in $M_{n}(\kappa)$ is a semisimple monoid.

Let $M$ be a reductive monoid with unit group $G$. The set $E(M)$ of idempotents of $M$ is partially ordered in the usual way:

$$
e \leq f \quad \text { if } \quad e f=e=f e
$$

By [6, Section 6] (or see [9, Chapter 9]) there is a cross-section $\Lambda$ of the $G \times G$-orbits $(\mathcal{J}$-classes) of $M$ consisting of idempotents so that for $e, f \in \Lambda$,

$$
e \leq f \Longleftrightarrow G e G \subseteq \overline{G f G} \Longleftrightarrow M e M \subseteq \overline{M f M}
$$

Here the closure is with respect to the Zariski topology. $\Lambda$ is a finite lattice and called the cross-section lattice of $M$. It is unique up to conjugacy by elements of $G$. We will let $\Lambda_{\min }$ denote the minimal elements of $\Lambda-\{0\}$ and $\Lambda_{\max }$ the maximal elements of $\Lambda-\{1\}$. All maximal chains in $\Lambda$ have the same length. This yields rank and corank functions on $\Lambda$ and hence on $M$ (via the decomposition of $M$ into $G \times G$-orbits):

$$
\mathrm{rk}: M \longrightarrow\{0, \ldots, n\}, \quad \text { crk }: M \longrightarrow\{0, \ldots, n\}
$$


where $n$ is the length of a maximal chain in $\Lambda$. Let

$$
\begin{array}{r}
B=B(\Lambda)=\{x \in G \mid x e=e x e \text { for all } e \in \Lambda\} \\
B^{-}=B^{-}(\Lambda)=\{x \in G \mid \text { ex }=\text { exe for all } e \in \Lambda\}
\end{array}
$$

Then $B, B^{-}$are opposite Borel subgroups of $G$ with maximal torus

$$
T=C_{G}(\Lambda)=\{x \in G \mid x e=e x \text { for all } e \in \Lambda\}
$$

and $\operatorname{dim} T$ is equal to the length of any maximal chain in $\Lambda$. The set $E(\bar{T})$ of 'diagonal idempotents' of $M$ is a finite lattice isomorphic to the face lattice of a polytope, with the set of vertices of the polytope corresponding to the set $E(\bar{T})_{\min }$ of minimal idempotents of $E(\bar{T})-\{0\}$.

Let $W=N_{G}(T) / T$ denote the Weyl group of $G$. Then

$$
E(\bar{T})=\bigcup_{x \in W} x \Lambda x^{-1}
$$

The set

$$
S=\{\alpha \in W \mid \alpha B \alpha \subseteq B \cup B \alpha B\}
$$

of simple reflections of $W$ generates $W$. We consider $S$ as an unlabeled Coxeter graph. So for $\alpha, \beta \in S, \alpha-\beta$ means $\alpha \beta \neq \beta \alpha$. If $S$ is connected, then we say that $\alpha \in S$ is an endpoint of $S$, if $S-\{\alpha\}$ is connected. If $S$ is connected and $\alpha \in S$, then since $W$ is finite, we see by the classification of Coxeter graphs that any component $S^{\prime}$ of $S-\{\alpha\}$ contains an endpoint of $S$ and also contains a unique $\gamma$ such that $\alpha-\gamma . G$ admits a Bruhat-decomposition with respect to $W$ :

$$
G=\bigsqcup_{w \in W} B w B
$$

The Bruhat-Chevalley order on $\mathrm{W}$ is given by [3]:

$$
x \leq y \quad \text { if } \quad B x B \subseteq \overline{B y B}, \quad x, y \in W
$$

Let $y \in W$. Then $y=\alpha_{1} \cdots \alpha_{m}$ for some $\alpha_{1}, \ldots, \alpha_{m} \in S$. If $m$ is minimal, then the length $l(y)$ of $y$ is defined to be $m$ and the support,

$$
\text { supp } y=\left\{\alpha_{1}, \ldots, \alpha_{m}\right\}
$$

If $\beta \in \operatorname{supp} y$, we say that $\beta$ appears in $y$. If $\beta \in S$ with $l(\beta y)<l(y)$, then by Matsumoto's exchange condition [4, 1.7],

$$
\beta y=\alpha_{1} \cdots \hat{\alpha_{i}} \cdots \alpha_{m} \text { for some } i
$$

For $x, y \in W$, let

$$
x * y= \begin{cases}x y & \text { if } l(x y)=l(x)+l(y) \\ \text { undefined } & \text { otherwise }\end{cases}
$$


If $\beta \in S, y \in W$, we will say that $\beta$ commutes with all of $y$ if $\alpha \beta=\beta \alpha$ for all $\alpha \in$ supp $y$. By [1, Lemma 1],

$$
\beta \notin \operatorname{supp} y, y \beta=\beta * y^{\prime} \Longrightarrow \beta \text { commutes with all of } y
$$

If $x, y \in W, y=\alpha_{1} \cdots \alpha_{m}, l(y)=m$, then it is known that the Bruhat-Chevalley order (2) has the following simple description:

$$
x \leq y \Longleftrightarrow x=\alpha_{i_{1}} \cdots \alpha_{i_{t}} \text {, for some } i_{1}<\cdots<i_{t}
$$

For $I \subseteq S, W_{I}=\langle I\rangle$ is called a parabolic subgroup of $W$. Let

$$
\begin{gathered}
D_{I}=\{x \in W \mid x \alpha=x * \alpha \forall \alpha \in I\}, \\
D_{I}^{-1}=\{x \in W \mid \alpha x=\alpha * x \forall \alpha \in I\} .
\end{gathered}
$$

Then

$$
W=D_{I} W_{I}=W_{I} D_{I}^{-1}
$$

We refer to [2, 4] for details. We are following here the notation in [2].

By [15] the Bruhat decomposition (1) of $G$ extends to $M$ :

$$
M=\bigsqcup_{\sigma \in R} B \sigma B
$$

where $R=\overline{N_{G}(T)} / T$ is the Renner monoid of $M . R$ is a finite inverse monoid with unit group $W$ and

$$
R=\bigsqcup_{e \in \Lambda} W e W
$$

Moreover $W e W, e \in \Lambda$, are the $\mathcal{J}$-classes ( $W \times W$-orbits) of $R$. For $M_{n}(\kappa), R$ is the monoid of all partial permutation matrices and called the rook monoid in combinatorics and the symmetric inverse semigroup in semigroup theory. For $e \in \Lambda$, let

$$
\lambda(e)=\{\alpha \in S \mid \alpha e=e \alpha\}
$$

and

$$
\lambda^{*}(e)=\bigcap_{f \geq e} \lambda(f), \quad \lambda_{*}(e)=\bigcap_{f \leq e} \lambda(f)
$$

Then

$$
\begin{array}{r}
W(e)=W_{\lambda(e)}=\{w \in W \mid w e=e w\}, \\
W^{*}(e)=W_{\lambda^{*}}(e), \\
W_{*}(e)=W_{\lambda_{*}(e)}=\{w \in W \mid w e=e=e w\}
\end{array}
$$

are parabolic subgroups of $W$ with

$$
W(e)=W^{*}(e) \times W_{*}(e)
$$


$W(e)$ is the Weyl group of $C_{G}(e), W^{*}(e)$ the Weyl group of the unit group $H(e)$ of $e M e$, and $W_{*}(e)$ the Weyl group of the identity component of

$$
G_{e}=\{g \in G \mid g e=e=e g\}
$$

Let

$$
\begin{gathered}
D(e)=D_{\lambda(e)}=\{y \in W \mid y \beta>y \text { for all } \beta \in \lambda(e)\}, \\
D(e)^{-1}=D_{\lambda(e)}^{-1}=\{y \in W \mid \beta y>y \text { for all } \beta \in \lambda(e)\}
\end{gathered}
$$

The Bruhat-Chevalley order (2) on W has a natural extension to $R$ :

$$
\sigma \leq \theta \text { if } B \sigma B \subseteq \overline{B \theta B}, \sigma, \theta \in R
$$

The order has been determined in [5] and more explicitly in [11, 12]. In particular for $e, e^{\prime} \in \Lambda, x, y \in W$,

$$
e \leq e^{\prime} \Longrightarrow x e y \leq x e^{\prime} y
$$

and for $y \in D(e)^{-1}, y^{\prime} \in D\left(e^{\prime}\right)^{-1}$,

$$
e y \leq e^{\prime} y^{\prime} \Longleftrightarrow y^{\prime} \leq z y \text { for some } z \in W(e)
$$

In particular for $y, y^{\prime} \in D(e)^{-1}$,

$$
e y \leq e y^{\prime} \Longleftrightarrow y^{\prime} \leq y
$$

In the study of reductive monoids, the cross-section lattice $\Lambda$ along with the type map

$$
\lambda: \Lambda \longrightarrow 2^{S}
$$

takes the place of the Dynkin diagram for a reductive group. This data along with the Weyl group determines the Renner monoid $R$, and along with $G$ determines the structure of the poset (or more precisely the biordered set) $E(M)$ of idempotents of $M$. In general the structure of $\Lambda$ is very complicated and not well understood. The exceptions are when $\left|\Lambda_{\text {min }}\right|=1$ ( $\mathcal{J}$-irreducible monoids) and when $\left|\Lambda_{\max }\right|=1$ ( $\mathcal{J}$-coirreducible monoids). We will discuss these monoids more in Sects. 5 and 6.

We will also need to consider another decomposition of $M$, related to conjugacy classes, studied by the author beginning in [8] and culminating in [10, 13]. First, following [15], let the set of Gauss-Jordan elements of $R$,

$$
G J=\left\{e y \mid e \in \Lambda, y \in D(e)^{-1}\right\}
$$

For $M_{n}(\kappa)$, this consists of partial permutation matrices in row-echelon form. For $e y, e^{\prime} y^{\prime} \in G J$, define $e y \sim e^{\prime} y^{\prime}$ if wey $w^{-1}=e^{\prime} y^{\prime}$ for some $w \in W$. This implies that $e=e^{\prime}$. Let $\mathcal{C}=G J / \sim$ and let [ey] denote the $\sim$-class of $e y$. Then by [13], $\mathcal{C}$ is a poset if we define:

$$
[e y] \leq\left[e^{\prime} y^{\prime}\right] \text { if } w e y w^{-1} \leq e^{\prime} y^{\prime} \text { for some } w \in W
$$


We call $\mathcal{C}$ the conjugacy poset of $M$, in that the associated decomposition (17) below leads to a description of the conjugacy classes of $M$. We note that in $[10,13,16], \mathcal{C}$ is denoted by $R^{*}, \tilde{R}$ and $P$, respectively. For $M_{n}(\kappa), \mathcal{C}$ is the poset of all partitions of $m, m \leq n$, with respect to a generalized dominance order. For $[e y] \in \mathcal{C}$, let

$$
X(e y)=\bigcup_{g \in G} g B e y B g^{-1}
$$

The conjugacy decomposition of $M$, obtained in [10, 13], is:

$$
M=\bigsqcup_{[e y] \in \mathcal{C}} X(e y)
$$

The conjugacy classes of $M$ in $X(e y)$ are in a natural 1-1 correspondence with the twisted conjugacy classes of a finite collection of reductive groups with automorphisms. For $[e y],\left[e^{\prime} y^{\prime}\right] \in \mathcal{C}$,

$$
X(e y) \subseteq \overline{X\left(e^{\prime} y^{\prime}\right)} \Longleftrightarrow[e y] \leq\left[e^{\prime} y^{\prime}\right]
$$

and for $[e y] \in \mathcal{C}$,

$$
\overline{X(e y)}=\bigsqcup_{\left[e^{\prime} y^{\prime}\right] \leq[e y]} X\left(e^{\prime} y^{\prime}\right)
$$

Now let $e \in \Lambda_{\max }, \lambda(e)=\lambda^{*}(e)=I$. Then for $y, y^{\prime} \in D_{I}^{-1}$, we have by [13, Theorem 2.9] that

$$
[e y] \leq\left[e y^{\prime}\right] \Longleftrightarrow w y^{\prime} \leq y w \text { for some } w \in W_{I}
$$

Then

$$
l(w)+l\left(y^{\prime}\right)=l\left(w y^{\prime}\right) \leq l(y w) \leq l(w)+l(y)
$$

So $l\left(y^{\prime}\right) \leq l(y)$. If further $l(y)=l\left(y^{\prime}\right)$, then $l\left(w y^{\prime}\right)=l(y w)$. This implies that $w * y^{\prime}=y * w$ and hence that $y=y^{\prime}$. Thus for $e \in \Lambda_{\max }$,

$$
[e y] \leq\left[e y^{\prime}\right] \Longrightarrow l\left(y^{\prime}\right) \leq l(y), \quad[e y]<\left[e y^{\prime}\right] \Longrightarrow l\left(y^{\prime}\right)<l(y)
$$

\section{Linear and Coxeter elements}

Let $W$ be a finite Weyl group with generating set $S$ of simple reflections. We will call $w \in W$ linear if $w=\alpha_{1} \cdots \alpha_{m}$ with $\alpha_{1}, \ldots, \alpha_{m} \in S$ distinct. If further $m=|S|$, then $w$ is called a Coxeter element. Thus linear elements of $W$ are Coxeter elements of parabolic subgroups of $W$. In this section we prove some technical results concerning linear and Coxeter elements that will be needed in our study of the nilpotent variety of a reductive monoid.

Lemma 3.1 Let $y=\alpha_{1} \cdots \alpha_{m}$ be linear, $\beta \in S, \beta y<y$. Then $\beta=\alpha_{j}$ for some $j$ and $\beta \alpha_{i}=\alpha_{i} \beta$ for $1 \leq i \leq j$. 
Proof If $\beta \neq \alpha_{j}$ for all $j$, then clearly $\beta y>y$. So $\beta=\alpha_{j}$ for some $j$. By the exchange condition (3), there exists $k$ such that

$$
\beta \alpha_{1} \cdots \alpha_{m}=\alpha_{1} \cdots \hat{\alpha_{k}} \cdots \alpha_{m}
$$

So

$$
\alpha_{1} \cdots \alpha_{k}=\beta \alpha_{1} \cdots \alpha_{k-1}
$$

So $\alpha_{k}=\beta$ and $k=j$. Hence

$$
\alpha_{1} \cdots \alpha_{j}=\alpha_{j} \alpha_{1} \cdots \alpha_{j-1}
$$

By (4), $\alpha_{i} \alpha_{j}=\alpha_{j} \alpha_{i}$ for $1 \leq i \leq j$.

Lemma 3.2 Let $S$ be connected, $\alpha \in S$. Then there is a unique Coxeter element $c_{S}(\alpha)$ of $W$ in $D_{S-\{\alpha\}}^{-1}$.

Proof We prove by induction on $|S|$. Let $S_{1}, \ldots, S_{t}, t \leq 3$, be the components of $S-\{\alpha\}$. Then there exist unique $\beta_{i} \in S_{i}, i=1, \ldots, t$ such that $\alpha-\beta_{i}$ and $y=$ $\alpha c_{S_{1}}\left(\beta_{1}\right) \cdots c_{S_{t}}\left(\beta_{t}\right)$ is a Coxeter element of $W$ and is in $D_{S-\{\alpha\}}^{-1}$ by Lemma 3.1.

Conversely let $y$ be a Coxeter element of $W$ in $D_{S-\{\alpha\}}^{-1}$. Then $\alpha y<y$. Let $y=$ $\alpha * y_{0}$. Then $y_{0}=y_{1} \cdots y_{t}$ where $y_{i}$ is a Coxeter element of $W_{S_{i}}$. We claim that $y_{i} \in D_{S_{i}-\left\{\beta_{i}\right\}}^{-1}$. Otherwise $\gamma y_{i}<y_{i}$ for some $\gamma \in S_{i}, \gamma \neq \beta_{i}$. Then $\gamma \alpha=\alpha \gamma$ and $\gamma$ commutes with all $y_{j}, j \neq i$. Hence

$$
\gamma y=\gamma \alpha y_{0}=\gamma \alpha y_{1} \cdots y_{t}=\alpha y_{1} \cdots \gamma y_{i} \cdots y_{t}<\alpha y_{1} \cdots y_{i} \cdots y_{t}=\alpha y_{0}=y,
$$

a contradiction since $y \in D_{S-\{\alpha\}}^{-1}$. Hence $y_{i} \in D_{S_{i}-\left\{\beta_{i}\right\}}^{-1}, i=1, \ldots, t$. By induction hypothesis, $y_{i}=c_{S_{i}}\left(\beta_{i}\right), i=1, \ldots, t$. Hence

$$
y=\alpha y_{0}=\alpha y_{1} \cdots y_{t}=\alpha c_{S_{1}}\left(\beta_{1}\right) \cdots c_{S_{t}}\left(\beta_{t}\right)
$$

This completes the proof.

Lemma 3.3 Let $I, K \subseteq S$ such that no component of $K$ is contained in I. Then there is a Coxeter element $z$ of $W_{K}$ in $D_{I}^{-1}$.

Proof Let $K_{1}, \ldots, K_{t}$ denote the components of $K$. Then there exists $\alpha_{i} \in K_{i}, \alpha_{i} \notin I$, $i=1, \ldots, t$. Let $z_{i}=c_{K_{i}}\left(\alpha_{i}\right), i=1, \ldots, t$ as in Lemma 3.2. We claim that $z_{i} \in D_{I}^{-1}$. Otherwise $\beta z_{i}<z_{i}$ for some $\beta \in I$. So $\beta \in$ supp $z_{i} \subseteq K_{i}$. Since $\beta \in I, \beta \neq \alpha_{i}$. So $z_{i} \notin D_{K_{i}-\left\{\alpha_{i}\right\}}^{-1}$, a contradiction. Thus $z_{i} \in D_{I}^{-1}, i=1, \ldots, t$. Clearly $z=z_{1} \cdots z_{t}$ is a Coxeter element of $W_{K}$. Also $z \in D_{I}^{-1}$ by Lemma 3.1. This completes the proof.

Lemma 3.4 Let $I \subseteq S, y \in D_{I}^{-1}$. Then there exists linear $y_{0} \in D_{I}^{-1}$ such that $y_{0} \leq y$ and $\operatorname{supp} y_{0}=\operatorname{supp} y$. 
Proof Let $K=\operatorname{supp} y$. We prove by induction on $l(y)$. Let $y=x * \alpha_{0}, \alpha_{0} \in K$. Then $x \in D_{I}^{-1}$. If $\alpha_{0}$ appears in $x$, we are done by the induction hypothesis. So assume that $\alpha_{0}$ does not appear in $x$. By the induction hypothesis, supp $x=\left\{\alpha_{1}, \ldots, \alpha_{m}\right\}$, $z=\alpha_{m} \cdots \alpha_{1} \in D_{I}^{-1}$. Then $y_{0}=z \alpha_{0} \leq y$. we claim that $z \alpha_{0} \in D_{I}^{-1}$. Otherwise for some $\beta \in I, \beta z \alpha_{0}<z \alpha_{0}$. Since $\beta z>z$, we see by Lemma 3.1 that $\beta=\alpha_{0}$ and that $\beta$ commutes with all of $z$. So $y=x * \alpha_{0}=\alpha_{0} * x$ and $\beta y=\alpha_{0} y=x<y$, a contradiction since $y \in D_{I}^{-1}$. Hence $y_{0}=z \alpha_{0} \in D_{I}^{-1}$. This completes the proof.

Lemma 3.5 Let $I \subseteq S, \alpha_{0} \in S-I, I^{\prime}$ a component of $S-\left\{\alpha_{0}\right\}$ contained in I. Let $\alpha_{1}, \ldots, \alpha_{k} \in I^{\prime}$ be distinct such that $\alpha_{0}-\alpha_{1}-\cdots-\alpha_{k}$. Then

(i) $y_{0}=\alpha_{0} \alpha_{1} \cdots \alpha_{k} \in D_{I}^{-1}$.

(ii) If $y \in D_{I}^{-1}$ is linear with $\alpha_{0} y<y$ and if $\alpha_{k}$ appears in $y$, then $y_{0} \leq y$.

Proof (i) Let $\beta \in I$ such that $\beta y_{0}<y_{0}$. Since $\alpha_{0} \notin I$, we see by Lemma 3.1 that $\beta=\alpha_{i}$ for some $1 \leq i \leq k$ and that $\alpha_{i} \alpha_{i-1}=\alpha_{i-1} \alpha_{i}$, a contradiction. Hence $y_{0} \in D_{I}^{-1}$.

(ii) We prove by induction on $l(y)$. Since $\alpha_{0} y<y$, we see that $y=\alpha_{0} * y_{1}$ and supp $y_{1} \subseteq S-\left\{\alpha_{0}\right\}$. So $y_{1}=y_{2} * y_{3}$ with $\alpha_{k} \in \operatorname{supp} y_{2} \subseteq I^{\prime}$. Hence $\alpha_{0} y_{2} \in D_{I}^{-1}$ and $\alpha_{0} y_{2} \leq y$. So by induction hypothesis we may assume that $\alpha_{0} y_{2}=y$. Hence $y_{1}=y_{2} \in W_{I^{\prime}}$. Since $W$ is finite, $\alpha_{0}$ commutes with all elements of $I^{\prime}$ other than $\alpha_{1}$. Since $y \in D_{I}^{-1}, y_{1}=\alpha_{1} * z$. Let $\gamma \in I^{\prime}, \gamma \neq \alpha_{1}, \gamma y_{1}<y_{1}$. Then

$$
\gamma y=\gamma \alpha_{0} y_{1}=\alpha_{0} \gamma y_{1}<\alpha_{0} y_{1}=y
$$

a contradiction since $y \in D_{I}^{-1}$. Hence $y_{1} \in D_{I_{1}}^{-1}$, where $I_{1}=I^{\prime}-\left\{\alpha_{1}\right\}$. Since $y_{1} \in W_{I^{\prime}}$, the induction hypothesis implies that $\alpha_{1} \cdots \alpha_{k} \leq y_{1}$. So $y_{0}=\alpha_{0} \alpha_{1} \cdots \alpha_{k} \leq$ $\alpha_{0} y_{1}=y$, completing the proof.

Lemma 3.6 Let $S$ be connected, $I \subsetneq S, y \in D_{I}^{-1}, y$ linear. Suppose that for any $\alpha \in S$ with no component of $S-\{\alpha\}$ contained in I, $\alpha$ appears in $y$. Then $y$ is a Coxeter element of $W$.

Proof If $I=\emptyset$, the result is clear. So let $I \neq \emptyset$. We prove by induction on $|S-I|$. First suppose that $|S-I|=1$ and $I=S-\left\{\alpha_{0}\right\}$. Let $\alpha \in I, I^{\prime}$ the component of $\alpha$ in $I$. Then

$$
\alpha_{0}-\alpha_{1}-\cdots-\alpha-\cdots-\alpha_{k}
$$

with $\alpha_{k}$ being an endpoint of $S$. Now $S-\left\{\alpha_{k}\right\}$ is connected and not contained in $I$. Hence $\alpha_{k}$ appears in $y$. Since $y \in D_{I}^{-1}, \alpha_{0} y<y$. By Lemma 3.5, $\alpha_{0}, \alpha$ appear in $y$. Hence $y$ is a Coxeter element of $W$.

Next assume that $|S-I|>1$. First suppose that $\beta y>y$ for some $\beta \in S-I$ and let $I_{1}=I \cup\{\beta\}$. The $I_{1} \neq S$ and $y \in D_{I_{1}}^{-1}$. Let $\alpha \in S$ such that no component of $S-\{\alpha\}$ is contained in $I_{1}$. Then no component of $S-\{\alpha\}$ is contained in $I$. Hence $\alpha$ appears in $y$. Since $\left|S-I_{1}\right|<|S-I|$, we see by the induction hypothesis that $y$ is a Coxeter element of $W$. Hence assume that $\beta y<y$ for all $\beta \in S-I$. Then $\beta$ appears 
in $y$ for all $\beta \in S-I$. Let $\alpha \in I$. If no component of $S-\{\alpha\}$ is contained in $I$, then $\alpha$ appears in $y$. So assume that some component $I^{\prime}$ of $S-\{\alpha\}$ is contained in $I$. Then $I^{\prime}$ contains an endpoint $\gamma$ of $S$ and

$$
\alpha=\alpha_{0}-\alpha_{1}-\cdots-\alpha_{k}=\gamma, \quad \alpha_{1}, \ldots, \alpha_{k} \in I^{\prime}
$$

Now $S-\{\gamma\}$ is connected and not contained in $I$. So $\gamma=\alpha_{k}$ appears in $y$. Since $\alpha \in I$, there exists $\beta \in S-I$ such that

$$
\beta=\beta_{0}-\beta_{1}-\cdots-\beta_{m}=\alpha \quad \text { with } \beta_{1}, \ldots, \beta_{m} \in I
$$

Let $I^{\prime \prime}$ be the component of $\beta$ in $S-\{\alpha\}$. Then since $I^{\prime} \subseteq I$ and $\beta \notin I, I^{\prime} \neq I^{\prime \prime}$. So $\beta_{0}, \ldots, \beta_{m-1} \in I^{\prime \prime}$ and

$$
\beta=\beta_{0}-\cdots-\beta_{m}-\alpha_{1}-\cdots-\alpha_{k}
$$

Since $\beta \notin I, \beta y<y$. Since also $\alpha_{k}$ appears in $y$, we see by Lemma 3.5 that $\alpha=\beta_{m}$ appears in $y$. Hence $y$ is a Coxeter element of $W$, completing the proof.

\section{Nilpotent variety}

For a reductive monoid $M$, the nilpotent variety

$$
M_{\text {nil }}=\left\{a \in M \mid a^{k}=0 \text { for some } k\right\}
$$

This closed subset of $M$ is in general not irreducible.

Example 4.1 Let

$$
M=\left\{A \otimes B \mid A, B \in M_{2}(\kappa)\right\}
$$

Then $M_{\text {nil }}$ has two components:

$$
\left\{A \otimes B \mid A^{2}=0\right\} \quad \text { and } \quad\left\{A \otimes B \mid B^{2}=0\right\}
$$

The variety $M_{n i l}$ is best studied by analyzing the conjugacy poset $\mathcal{C}$. Let

$$
\mathcal{C}_{\text {nil }}=\left\{[e y] \in \mathcal{C} \mid(e y)^{k}=0 \text { for some } k\right\}
$$

The following result is proved in [10, Theorem 3.1(i)]. We include a proof here since the proof given in [10] needs minor elaboration.

Theorem 4.2 Let $0 \neq e \in \Lambda, y \in D(e)^{-1}$. Then $[e y] \in \mathcal{C}_{\text {nil }}$ if and only if supp $y \nsubseteq$ $\lambda(f)$ for all $f \in \Lambda_{\text {min }}$ with $f \leq e$.

Proof If $N=|W|$, then by [10, Equation (32)],

$$
f_{0}=(e y)^{N}=\prod_{i} y^{i} e y^{-i} \in \Lambda
$$


Then $f_{0} \leq e$ and

$$
y \in W\left(f_{0}\right)=W^{*}\left(f_{0}\right) W_{*}\left(f_{0}\right) \subseteq W^{*}(e) W_{*}\left(f_{0}\right)
$$

Since $y \in D(e)^{-1}, y \in W_{*}\left(f_{0}\right)$. So if $e y$ is not nilpotent, then $f_{0} \neq 0$ and $f_{0} \geq f$ for some $f \in \Lambda_{\min }$. So $e \geq f$ and

$$
\text { supp } y \subseteq \lambda_{*}\left(f_{0}\right) \subseteq \lambda_{*}(f)=\lambda(f)
$$

Conversely suppose that supp $y \subseteq \lambda(f)$ for some $f \in \Lambda_{\min }$ with $e \geq f$. Then $y \in$ $W(f)=W_{*}(f)$ and $y f=f$. Hence $f_{0} f=(e y)^{N} f=f$ and $f_{0} \geq f$. So $[e y] \notin \mathcal{C}_{\text {nil }}$. This completes the proof.

The following result is proved in [10, Theorem 3.1(ii),(iii)].

Theorem 4.3 The variety $M_{\text {nil }}$ decomposes as:

$$
M_{n i l}=\bigsqcup_{[e y] \in \mathcal{C}_{n i l}} X(e y)
$$

The irreducible components of $M_{\text {nil }}$ are $\overline{X(e y)}$, where [ey] is a maximal element of $\mathcal{C}_{\text {nil }}$ with respect to the order (15).

The open problem then is to determine the maximal elements of $\mathcal{C}_{\text {nil }}$. We will solve this problem for $\mathcal{J}$-irreducible and $\mathcal{J}$-correducible monoids in Sects. 5 and 6 , respectively. We end this section with the following auxiliary result for semisimple monoids.

Theorem 4.4 Let $M$ be a semisimple monoid. Then $M_{\text {nil }} \cap J \neq \emptyset$ for all $\mathcal{J}$-classes $J \neq G$ of $M$.

Proof Let $e \in \Lambda, e \neq 0,1$. If $w_{0}$ and $v_{0}$ are the respective maximum elements of $W$ and $W(e)$, then $x=v_{0} w_{0}$ is the maximum element of $D(e)^{-1}$. Let $f \in \Lambda_{\text {min }}$, $e \geq f$. Suppose $x \in W(f)$. Then for all $y \in D(e)^{-1}, y \leq x$ and hence $y \in W(f)$. So $D(e)^{-1} \subseteq W(f)$ and by (6),

$$
W=W(e) D(e)^{-1} \subseteq W(e) W(f)
$$

Let $w \in W$. Then $w=w_{1} w_{2}, w_{1} \in W(e), w_{2} \in W(f)$. So

$$
w^{-1} e w=w_{2}^{-1} w_{1}^{-1} e w_{1} w_{2}=w_{2}^{-1} e w_{2} \geq w_{2}^{-1} f w_{2}=f
$$

Hence

$$
e^{\prime}=\prod_{w \in W} w^{-1} e w \geq f
$$

Clearly $w e^{\prime}=e^{\prime} w$ for all $w \in W$. By [9, Theorem 6.30], $e^{\prime} \in \overline{Z(G)}$. Since $e^{\prime} \neq 1$ and $M$ is semisimple, $e^{\prime}=0$. Hence $f=0$, a contradiction. So $x \notin W(f)$. By Theorem 4.2, $[e x] \in \mathcal{C}_{\text {nil }}$. So $M_{\text {nil }} \cap G e G \neq \emptyset$, proving the theorem. 


\section{$5 \mathcal{J}$-irreducible monoids}

If $G_{0}$ is a semisimple group and $\theta: G_{0} \rightarrow G L_{n}(\kappa)$ an irreducible representation, then $\overline{\kappa \theta\left(G_{0}\right)}$ is a reductive monoid with a unique non-zero minimal $\mathcal{J}$-class. Call a reductive monoid $M, \mathcal{J}$-irreducible, if $\left|\Lambda_{\min }\right|=1$. Let $\Lambda_{\min }=\left\{e_{0}\right\}$. Then $J_{0}=$ $G e_{0} G$ is the unique non-zero minimal $\mathcal{J}$-class of $M$. Let $\lambda\left(e_{0}\right)=I$. We then say that $M$ is a $\mathcal{J}$-irreducible monoid of type $I$. Now no component of $S$ is contained in $I$. For suppose some component $S^{\prime}$ of $S$ is contained in $I$. Then $W=W_{S^{\prime}} \times W_{S^{\prime \prime}}$. Let $e \in E(\bar{T})_{\min }$. Then $x^{-1} e_{0} x=e$ for some $x \in W$. Since $W_{S^{\prime}} \subseteq W\left(e_{0}\right), w^{-1} e_{0} w=e$ for some $w \in W_{S^{\prime \prime}}$. So for $u \in W_{S^{\prime}}$,

$$
u^{-1} e u=u^{-1} w^{-1} e_{0} w u=w^{-1} u^{-1} e_{0} u w=w^{-1} e_{0} w=e
$$

If $f \in E(\bar{T}), f \neq 0$, then $f$ is the join of some elements in $E(\bar{T})_{\text {min }}$. Hence $u^{-1} f u=f$. So by [9, Chapter 8], $u=1$. Hence $S^{\prime}=\emptyset$, a contradiction. Thus no component of $S$ is contained in $I$. Let $S_{1}, \ldots, S_{t}$ be the components of $S, I_{j}=S_{j} \cap I$, $j=1, \ldots, t$. Then $\left|I_{j}\right|<\left|S_{j}\right|, j=1, \ldots, t$. We say that $M$ is $\mathcal{J}$-irreducible of maximal type if $\left|I_{j}\right|=\left|S_{j}\right|-1, j=1, \ldots, t$. This concept is not used in this paper, but its dual concept is used in Sect. 6. Rather amazingly, the cross-section lattice $\Lambda$ and the type map $\lambda$ of a $\mathcal{J}$-irreducible monoid are completely determined its type $I$. The following result is proved in [14].

\section{Theorem 5.1 Let $M$ be a $\mathcal{J}$-irreducible monoid of type I. Then}

(i) $M$ is semisimple.

(ii) If $e, e^{\prime} \in \Lambda-\{0\}$, then $e \leq e^{\prime}$ if and only if $\lambda^{*}(e) \subseteq \lambda^{*}\left(e^{\prime}\right)$.

(iii) If $e \in \Lambda-\{0\}$, then $\lambda_{*}(e)=\left\{\alpha \in I \mid \alpha \beta=\beta \alpha\right.$ for all $\left.\beta \in \lambda^{*}(e)\right\}$.

(iv) If $K \subseteq S$, then $K=\lambda^{*}(e)$ for some $e \in \Lambda-\{0\}$ if and only if no component of $K$ is contained in $I$.

(v) If $e \in \Lambda-\{0\}$, then $\left|\lambda^{*}(e)\right|=r k(e)-1=|S|-$ crk (e). In particular if $e \in \Lambda_{\text {max }}$, then $\lambda(e)=\lambda^{*}(e)=S-\{\alpha\}$ for some $\alpha \in S$.

We now proceed to determine the irreducible components of $M_{\text {nil }}$ for a $\mathcal{J}$-irreducible monoid $M$ of type $I$. We begin with:

Theorem 5.2 Let $M$ be a $\mathcal{J}$-irreducible monoid. Then every component of $M_{\text {nil }}$ intersects a unique maximal $\mathcal{J}$-class $J \neq G$ of $M$.

Proof We only need to prove existence of the maximal $\mathcal{J}$-class, since the uniqueness then follows from Theorem 4.3. We proceed by induction on $|S|$. Let $0 \neq[e y] \in \mathcal{C}_{\text {nil }}$. By Theorem 4.2, $y \notin W_{I}$. If $e \in \Lambda_{\text {max }}$, there is nothing to prove. So assume $e \notin$ $\Lambda_{\text {max }}$. Since $y \notin W_{I}, y=y_{1} * w$ with $w \in W_{I}, 1 \neq y_{1} \in D(e)^{-1} \cap D_{I}$. Then by (14), ey $\leq e y_{1}$ and by Theorem $4.2,\left[e y_{1}\right] \in \mathcal{C}_{\text {nil }}$. Thus we may assume that $y=y_{1} \in$ $D(e)^{-1} \cap D_{I}$. Let $f \in \Lambda_{\max }, e<f$. Suppose $y \notin W(f)$. Then $y=w * y^{\prime}$ with $w \in W(f)$ and $1 \neq y^{\prime} \in D(f)^{-1} \cap D_{I}$. So by (13), ey $\leq f y^{\prime}$ and by Theorem 4.2, $f y^{\prime} \in \mathcal{C}_{\text {nil }}$. So assume that $y \in W(f)$ for all $f \in \Lambda_{\max }$ with $e<f$. Let $f \in \Lambda_{\text {max }}$ with $e<f$. Then applying induction hypothesis on the $\mathcal{J}$-irreducible monoid $f M f$, 
we see that $[e y] \leq\left[e_{1} z\right]$ with $\left[e_{1} z\right] \in \mathcal{C}_{\text {nil }}$ and $f$ covering $e_{1}$ in $\Lambda$. Thus we may assume that $f$ covers $e$ in $\Lambda$. Then by Theorem 5.1, $\lambda(f)=\lambda^{*}(f)=S-\{\alpha\}, \lambda^{*}(e)=$ $S-\{\alpha, \beta\}$. If $S-\{\beta\}$ has no components contained in $I$, then by Theorem 5.1 , there exists $f^{\prime} \in \Lambda_{\text {max }}$ such that $\lambda\left(f^{\prime}\right)=\lambda^{*}\left(f^{\prime}\right)=S-\{\beta\}$. Then $e<f^{\prime}$ and $f f^{\prime}=e$. So

$$
\text { supp } y \in \lambda(f) \cap \lambda\left(f^{\prime}\right) \subseteq \lambda\left(f f^{\prime}\right)=\lambda(e)
$$

and $y \in W(e)$, a contradiction. So $S-\{\beta\}$ has a component contained in $I$. Since $S-\{\alpha, \beta\}=\lambda^{*}(e)$ has no components contained in $I$, it follows that $\{\alpha\}$ is a component of $S-\{\beta\}$ and contained in $I$. Thus $\lambda_{*}(e)=\{\alpha\}$ and $\lambda(e)=S-\{\beta\}$. So $\alpha \gamma=\gamma \alpha$ for all $\gamma \in S-\{\beta\}$. If also $\alpha \beta=\beta \alpha$, then $\{\alpha\}$ is a component of $S$ contained in $I$, a contradiction. Hence $\alpha \beta \neq \beta \alpha$. Since $1 \neq y \in D(e)^{-1}$ and $\lambda(e)=S-\{\beta\}$, we see that $y=\beta * y_{0}$. Let

$$
\alpha y=\alpha * y=w * x, w \in W(f), x \in D(f)^{-1}
$$

Then by (13), ey $\leq f x$. So if $[f x] \in \mathcal{C}_{\text {nil }}$, we are done. So assume that $f x$ is not nilpotent. Then by Theorem 4.2, $x \in W_{I}$. By (22), $\alpha(w x)=y<w x$. Also since $W(f)=S-\{\alpha\}, \alpha$ does not appear in $w$. So by the exchange condition (3),

$$
y=\alpha w x=w * x^{\prime}, x^{\prime}<x, l\left(x^{\prime}\right)=l(x)-1
$$

So $x^{\prime} \in W_{I}$. Since $y \in D_{I}, x^{\prime}=1$. So $l(x)=1$. Since $\alpha$ does not appear in $w$, we see by (22) that $x=\alpha$. Hence $w \alpha=\alpha y$ and by (4), $\alpha$ commutes with all of $w$. Since $\beta$ appears in $y, \beta$ appears in $w$. Hence $\alpha \beta=\beta \alpha$, a contradiction. This completes the proof.

For a maximal $\mathcal{J}$-class $J \neq G$ of $M$, we proceed to determine precisely the components of $M_{\text {nil }}$ that intersect $J$. Let $\alpha \in S, S_{\alpha}$ the component of $\alpha$ in $S$. Then $S_{\alpha} \nsubseteq I=\lambda\left(e_{0}\right)$. Let $\mathcal{A}(I, \alpha)$ denote the set of all linear elements $y=\alpha_{0} \cdots \alpha_{k}$ of $S$ such that

$$
\alpha=\alpha_{0}, \alpha_{0}-\alpha_{1}-\cdots-\alpha_{k}, \alpha_{k} \notin I, \alpha_{i} \in I \text { for } i<k
$$

Thus

$$
\mathcal{A}(I, \alpha)=\{\alpha\} \Longleftrightarrow \alpha \notin I
$$

Since $S_{\alpha}-\{\alpha\}$ has at most 3 components, we see by the classification of Coxeter graphs of finite Weyl groups that

$$
1 \leq|\mathcal{A}(I, \alpha)| \leq 3
$$

Theorem 5.3 Let $M$ be a $\mathcal{J}$-irreducible monoid, $e \in \Lambda_{\max }, J=G e G, \lambda(e)=$ $S-\{\alpha\}$. Then $\mathcal{A}(I, \alpha) \subseteq D(e)^{-1}$ and the distinct irreducible components of $M_{\text {nil }}$ that intersect $J$ are $\overline{X(e y)}, y \in \mathcal{A}(I, \alpha)$. In particular, at most 3 components of $M_{\text {nil }}$ intersect $J$. 
Proof If $y_{0} \in \mathcal{A}(I, \alpha)$, then $y_{0} \in D(e)^{-1}$ by Lemma 3.5(i), and $\left[e y_{0}\right] \in \mathcal{C}_{\text {nil }}$ by Theorem 4.2. Let $[e y] \in \mathcal{C}_{\text {nil }}$ be maximal with respect to the order (15). Then by (14), Lemma 3.4, and Theorem 4.2, $y$ is linear. Let $S_{\alpha}$ denote the component of $\alpha$ in $S$. Since $y \in D(e)^{-1}$, supp $y \subseteq S_{\alpha}$. Let $\beta \in \operatorname{supp} y, \beta \notin I, y=y_{1} * \beta * y_{2}$. Then $y_{1}, y_{1} \beta \in D(e)^{-1}$. By (14) and the maximality of $[e y], y=y_{1} \beta$ and $y_{1} \in W_{I}$. Since $y \in D(e)^{-1}, \alpha y<y$. There exist distinct $\alpha=\alpha_{0}, \ldots, \alpha_{k}=\beta \in S$ such that $\alpha_{0}-\alpha_{1}-\cdots-\alpha_{k}$. By (14), the maximality of [ey], and Lemma 3.5(ii), $y=\alpha_{0} \alpha_{1} \cdots \alpha_{k}$. Hence $\alpha_{j} \in I$ for $j<k$. So $y \in \mathcal{A}(I, \alpha)$.

Let $y, y^{\prime} \in \mathcal{A}(I, \alpha),\left[e y^{\prime}\right]<[e y]$. Then by (23), $\alpha \in I$. There exist distinct $\alpha_{0}, \ldots, \alpha_{k} \in S$ and distinct $\alpha_{0}^{\prime}, \ldots, \alpha_{l}^{\prime} \in S$ such that

$$
\alpha=\alpha_{0}=\alpha_{0}^{\prime}, \quad \alpha_{k}, \alpha_{l}^{\prime} \notin I, \quad \alpha_{i}, \alpha_{j}^{\prime} \in I \text { for } i<k, j<l
$$

and

$$
\begin{aligned}
& y=\alpha_{0} \alpha_{1} \cdots \alpha_{k}, \quad \alpha_{0}-\alpha_{1}-\cdots-\alpha_{k} \\
& y^{\prime}=\alpha_{0}^{\prime} \alpha_{1}^{\prime} \cdots \alpha_{l}^{\prime}, \quad \alpha_{0}^{\prime}-\alpha_{1}^{\prime}-\cdots-\alpha_{l}^{\prime}
\end{aligned}
$$

We prove by induction on $k$ that this is not possible. By (20), there exists $w \in W(e)$ such that $w y \leq y^{\prime} w$. So $\alpha$ does not appear in $w$ and

$$
w *\left(\alpha \alpha_{1} \cdots \alpha_{k}\right) \leq \alpha \alpha_{1}^{\prime} \cdots \alpha_{l}^{\prime} w
$$

Hence $w \alpha \alpha_{1} \cdots \alpha_{k} \not \leq \alpha_{1}^{\prime} \cdots \alpha_{l}^{\prime} w$. Thus

$$
\alpha w \alpha \alpha_{1} \cdots \alpha_{k} \leq \alpha_{1}^{\prime} \cdots \alpha_{l}^{\prime} w
$$

Hence $\alpha$ does not appear in $\alpha w \alpha \alpha_{1} \cdots \alpha_{k}$. Thus

$$
\alpha\left(w \alpha \alpha_{1} \cdots \alpha_{k}\right)<w \alpha \alpha_{1} \cdots \alpha_{k}
$$

By the exchange condition (3), $\alpha w \alpha=w$. So $w * \alpha=\alpha * w$ and by (4), $\alpha$ commutes with all of $w$. Since $\alpha_{1} \alpha \neq \alpha \alpha_{1}, \alpha_{1}$ does not appear in $w$. So by (25),

$$
w *\left(\alpha_{1} \cdots \alpha_{k}\right) \leq \alpha_{1}^{\prime} \cdots \alpha_{l}^{\prime} w
$$

Since $\alpha_{1}$ does not appear in $w, \alpha_{1}=\alpha_{j}^{\prime}$ for some $j$. So $\alpha=\alpha_{0}^{\prime}-\alpha_{j}^{\prime}$ and $j=1$. By (26) and the induction hypothesis, $\alpha_{1} \cdots \alpha_{k}=\alpha_{1}^{\prime} \cdots \alpha_{l}^{\prime}$. Hence $y=y^{\prime}$, a contradiction. Thus $[e y], y \in \mathcal{A}(I, \alpha)$ are distinct and maximal in $\mathcal{C}_{\text {nil }}$. This completes the proof.

Example 5.4 Let

$$
G_{0}=\left\{A \otimes\left(A^{-1}\right)^{t} \mid A \in S L_{3}(\kappa)\right\}
$$

and let $M=\overline{\kappa G_{0}}$. See [9, Example 10.3]. Then $S=\{\alpha, \beta\}$ where $\alpha=(12)$ and $\beta=$ (2 3). $M$ is a $\mathcal{J}$-irreducible monoid of type $\emptyset$ and $\Lambda_{\max }=\left\{e_{\alpha}, e_{\beta}\right\}$ where $\lambda\left(e_{\alpha}\right)=$ $S-\{\alpha\}=\{\beta\}$ and $\lambda\left(e_{\beta}\right)=S-\{\beta\}=\{\alpha\}$. The irreducible components of $M_{\text {nil }}$ are:

$$
\overline{X\left(e_{\alpha} \alpha\right)}, \overline{X\left(e_{\beta} \beta\right)}
$$


corresponding to the elements $A \otimes B$ of $M_{\text {nil }}$ with rk $B \leq 1$ and rk $A \leq 1$, respectively.

Example 5.5 Let $S=\{\alpha, \beta, \gamma, \delta\}$ be of type $D_{4}$ with $\beta, \gamma, \delta$ being the endpoints:

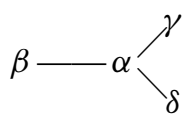

Let $I \subseteq S$ and consider a $\mathcal{J}$-irreducible monoid $M$ of Type $I$. Then by Theorem 5.1, $\Lambda_{\text {max }}$ consists of those $e_{\pi}, \pi \in S$, with $\lambda\left(e_{\pi}\right)=S-\{\pi\}$ having no components contained in $I$.

(i) If $I=\emptyset$, then $\Lambda_{\max }=\left\{e_{\alpha}, e_{\beta}, e_{\gamma}, e_{\delta}\right\}$ and $M_{\text {nil }}$ has 4 irreducible components:

$$
\overline{X\left(e_{\alpha} \alpha\right)}, \overline{X\left(e_{\beta} \beta\right)}, \overline{X\left(e_{\gamma} \gamma\right)}, \overline{X\left(e_{\delta} \delta\right)}
$$

(ii) If $I=\{\alpha\}$, then $\Lambda_{\max }=\left\{e_{\alpha}, e_{\beta}, e_{\gamma}, e_{\delta}\right\}$ and $M_{\text {nil }}$ has 6 irreducible components:

$$
\overline{X\left(e_{\alpha} \alpha \beta\right)}, \overline{X\left(e_{\alpha} \alpha \gamma\right)}, \overline{X\left(e_{\alpha} \alpha \delta\right)}, \overline{X\left(e_{\beta} \beta\right)}, \overline{X\left(e_{\gamma} \gamma\right)}, \overline{X\left(e_{\delta} \delta\right)}
$$

(iii) If $I=\{\beta\}$, then $\Lambda_{\max }=\left\{e_{\beta}, e_{\gamma}, e_{\delta}\right\}$ and $M_{\text {nil }}$ has 3 irreducible components:

$$
\overline{X\left(e_{\gamma} \gamma\right)}, \overline{X\left(e_{\delta} \delta\right)}, \overline{X\left(e_{\beta} \beta \alpha\right)}
$$

(iv) If $I=\{\alpha, \beta\}$, then $\Lambda_{\max }=\left\{e_{\beta}, e_{\gamma}, e_{\delta}\right\}$ and $M_{\text {nil }}$ has 4 irreducible components:

$$
\overline{X\left(e_{\gamma} \gamma\right)}, \overline{X\left(e_{\delta} \delta\right)}, \overline{X\left(e_{\beta} \beta \alpha \gamma\right)}, \overline{X\left(e_{\beta} \beta \alpha \delta\right)}
$$

(v) If $I=\{\beta, \gamma\}$, then $\Lambda_{\max }=\left\{e_{\beta}, e_{\gamma}, e_{\delta}\right\}$ and $M_{\text {nil }}$ has 3 irreducible components:

$$
\overline{X\left(e_{\beta} \beta \alpha\right)}, \overline{X\left(e_{\gamma} \gamma \alpha\right)}, \overline{X\left(e_{\delta} \delta\right)}
$$

(vi) If $I=\{\alpha, \beta, \gamma\}$, then $\Lambda_{\max }=\left\{e_{\beta}, e_{\gamma}\right\}$ and $M_{\text {nil }}$ has 2 irreducible components:

$$
\overline{X\left(e_{\beta} \beta \alpha \delta\right)}, \overline{X\left(e_{\gamma} \gamma \alpha \delta\right)}
$$

(vii) If $I=\{\beta, \gamma, \delta\}$, then $\Lambda_{\max }=\left\{e_{\beta}, e_{\gamma}, e_{\delta}\right\}$ and $M_{\text {nil }}$ has 3 irreducible components:

$$
\overline{X\left(e_{\beta} \beta \alpha\right)}, \overline{X\left(e_{\gamma} \gamma \alpha\right)}, \overline{X\left(e_{\delta} \delta \alpha\right)}
$$

Example 5.6 Let $M$ be a $\mathcal{J}$-irreducible monoid of type $\emptyset$. Such a monoid is called a canonical monoid and is of considerable importance in embedding theory, cf. [16]. For $\alpha \in S$, let $e_{\alpha} \in \Lambda$ be such that $\lambda\left(e_{\alpha}\right)=S-\{\alpha\}$. Then the components of $M_{\text {nil }}$ are $\overline{X\left(e_{\alpha} \alpha\right)}, \alpha \in S$. 


\section{$6 \mathcal{J}$-coirreducible monoids}

Call a reductive monoid $M, \mathcal{J}$-coirreducible, if $\left|\Lambda_{\max }\right|=1$. Let $\Lambda_{\max }=\left\{e_{0}\right\}$, $\lambda\left(e_{0}\right)=I$. We then say that $M$ is $\mathcal{J}$-coirreducible of type $I$. Then by the dual of the argument given in the beginning of Section 5, no component of $S$ is contained in $I$. Let $S_{1}, \ldots, S_{t}$ be the components of $S, I_{j}=S_{j} \cap I, j=1, \ldots, t$. Then $\left|I_{j}\right|<\left|S_{j}\right|$, $j=1, \ldots, t$. We say that $M$ is $\mathcal{J}$-coirreducible of maximal type if $\left|I_{j}\right|=\left|S_{j}\right|-1$, $j=1, \ldots, t$. The proof of Theorem 5.1 given in [14] dualizes to yield the following result.

Theorem 6.1 Let $M$ be a $\mathcal{J}$-coirreducible monoid of type I. Then

(i) $M$ is semisimple.

(ii) If $e, e^{\prime} \in \Lambda-\{1\}$, then $e \leq e^{\prime}$ if and only if $\lambda_{*}\left(e^{\prime}\right) \subseteq \lambda_{*}(e)$.

(iii) If $e \in \Lambda-\{1\}$, then $\lambda^{*}(e)=\left\{\alpha \in I \mid \alpha \beta=\beta \alpha \forall \beta \in \lambda_{*}(e)\right\}$.

(iv) If $K \subseteq S$, then $K=\lambda_{*}(e)$ for some $e \in \Lambda-\{1\}$ if and only if no component of $K$ is contained in $I$.

(v) If $e \in \Lambda-\{1\}$, then $\lambda_{*}(e)=\operatorname{crk}(e)-1=|S|-r k(e)$. In particular if $e \in \Lambda_{\text {min }}$, then $\lambda(e)=\lambda_{*}(e)=S-\{\alpha\}$ for some $\alpha \in S$.

We now describe the irreducible components of $M_{\text {nil }}$ for a $\mathcal{J}$-coirreducible monoid.

Theorem 6.2 Let $M$ be a $\mathcal{J}$-coirreducible monoid of type I. Then the distinct irreducible components of $M_{\text {nil }}$ are $\overline{X\left(e_{0} x\right)}$ where $x$ is a Coxeter element of $W$ in $D_{I}^{-1}$.

Proof Let $[e y] \in \mathcal{C}_{\text {nil }}$. We first show that $[e y] \leq\left[e_{0} x\right]$ for some $\left[e_{0} x\right] \in \mathcal{C}_{\text {nil }}$. By (14), Lemma 3.4, and Theorem 4.2, we may assume that $y$ is linear. Let $\lambda_{*}(e)=K$ and let

$$
y=y_{1} * w_{1} * y_{2} * w_{2} * \cdots * y_{m} * w_{m}, w_{i} \in W_{K}, y_{i} \in W_{S-K}
$$

Let $y^{\prime}=y_{1} y_{2} \cdots y_{m}$. We claim that $y^{\prime} \in D(e)^{-1}$. Otherwise there exists $\alpha \in \lambda(e)$ such that $\alpha y^{\prime}<y^{\prime}$. Then $\alpha \in \operatorname{supp} y^{\prime} \subseteq S-K$. So $\alpha \in \lambda(e)-\lambda_{*}(e)=\lambda^{*}(e)$. Since $\alpha y^{\prime}<y^{\prime}$ and $y^{\prime}$ is linear, we see by Lemma 3.1 that $\alpha y_{i}<y_{i}$ for some $i$ and that $\alpha$ commutes with all of $y_{j}$ for $j<i$. Since $\alpha \in \lambda^{*}(e), \alpha \beta=\beta \alpha$ for all $\beta \in \lambda_{*}(e)=K$. So $\alpha$ commutes with all of $w_{j}, j<i$. It follows that $\alpha y<y$, a contradiction since $y \in D(e)^{-1}$. Thus $y^{\prime} \in D(e)^{-1}$.

By Theorem 6.1, no component of $K$ is contained in $I$. So by Lemma 3.3, there is a Coxeter element $z$ of $W_{K}$ in $D_{I}^{-1}$. Let $x=z * y^{\prime}$. Then $x$ is linear. We claim that $x \in D_{I}^{-1}$. Let $\alpha \in I$ such that $\alpha x<x$. Since $z \in D_{I}^{-1}, \alpha z>z$. Since $x$ is linear, we see by Lemma 3.1 that $\alpha y^{\prime}<y^{\prime}$ and that $\alpha$ commutes with all of $z$. Since $z$ is a Coxeter element of $W_{K}, \alpha \beta=\beta \alpha$ for all $\beta \in K=\lambda_{*}(e)$. By Theorem 6.1(iii), $\alpha \in \lambda^{*}(e) \subseteq$ $\lambda(e)$. This is a contradiction since $y^{\prime} \in D(e)^{-1}$. Hence $x \in D_{I}^{-1}$. Next let $f \in \Lambda_{\text {min }}$. Then by Theorem 6.1(v), $\lambda(f)=\lambda_{*}(f)=S-\{\beta\}$ for some $\beta \in S$. First suppose $\beta \notin$ $K=\lambda_{*}(e)$. Then $\lambda_{*}(e) \subseteq \lambda *(f)$ and by Theorem 6.1(ii), $f \leq e$. Since $[e y] \in \mathcal{C}_{\text {nil }}$, we see by Theorem 4.2 that $\beta \in \operatorname{supp} y$. Since $\beta \notin K, \beta \in \operatorname{supp} y^{\prime} \subseteq \operatorname{supp} x$. Next 
let $\beta \in K$. Then since $z$ is a Coxeter element of $W_{K}, \beta \in \operatorname{supp} z \subseteq \operatorname{supp} x$. Thus in all cases $x \notin W(f)$. So by Theorem 4.2, [e $x] \in \mathcal{C}_{\text {nil }}$. Also by (13), (14),

$$
e y \leq e y^{\prime} \leq e_{0} x
$$

So $[e y] \leq\left[e_{0} x\right]$.

Now let $\left[e_{0} x\right]$ be a maximal element of $\mathcal{C}_{\text {nil }}$ with respect to the order (15). Then by (14), Lemma 3.4, and Theorem 4.2, $x$ is linear. Let $\alpha \in S$ such that no component of $S-\{\alpha\}$ is contained in $I$. Then by Theorem 6.1, there exists $f \in \Lambda_{\text {min }}$ such that $\lambda(f)=S-\{\alpha\}$. By Theorem 4.2, $\alpha$ appears in $x$. By Lemma 3.6, $x$ is a Coxeter element of $W$. Conversely if $x$ is a Coxeter element of $W$ in $D_{I}^{-1}$, then $\left[e_{0} x\right] \in \mathcal{C}_{\text {nil }}$ by Theorem 4.2. Finally if $x, x^{\prime}$ are Coxeter elements of $W$ in $D_{I}^{-1}$ and if $\left[e_{0} x\right] \leq\left[e_{0} x^{\prime}\right]$, then $x=x^{\prime}$ by (21). This completes the proof.

Example 6.3 Let

$$
G_{0}=\left\{A \oplus\left(A^{-1}\right)^{t} \mid A \in S L_{3}(\kappa)\right\}
$$

and let $M=\overline{\kappa G_{0}}$. See [9, Example 10.4]. Then $S=\{\alpha, \beta\}$ where $\alpha=\left(\begin{array}{ll}1 & 2) \text { and }\end{array}\right.$ $\beta=(23) . M$ is a $\mathcal{J}$-coirreducible monoid of type $\emptyset$. If $\Lambda_{\max }=\left\{e_{0}\right\}$, then the irreducible components of $M_{n i l}$ are $\overline{X\left(e_{0} \alpha \beta\right)}$ and $\overline{X\left(e_{0} \beta \alpha\right)}$.

Example 6.4 Let $S=\{\alpha, \beta, \gamma, \delta\}$ be of type $D_{4}$ with $\beta, \gamma, \delta$ being the endpoints:<smiles></smiles>

Let $I \subseteq S$ and consider a $\mathcal{J}$-coirreducible $M$ of type $I$. Let $\Lambda_{\max }=\left\{e_{0}\right\}$.

(i) If $I=\emptyset$, then $M_{\text {nil }}$ has 8 irreducible components:

$$
\begin{gathered}
\overline{X\left(e_{0} \beta \alpha \gamma \delta\right)}, \overline{X\left(e_{0} \gamma \alpha \beta \delta\right)}, \overline{X\left(e_{0} \delta \alpha \beta \gamma\right)}, \overline{X\left(e_{0} \beta \gamma \alpha \delta\right)}, \\
\overline{X\left(e_{0} \beta \delta \alpha \gamma\right)}, \overline{X\left(e_{0} \gamma \delta \alpha \beta\right)}, \overline{X\left(e_{0} \beta \gamma \delta \alpha\right)}, \overline{X\left(e_{0} \alpha \beta \gamma \delta\right)}
\end{gathered}
$$

(ii) If $I=\{\alpha\}$, then $M_{\text {nil }}$ has 7 irreducible components:

$$
\begin{array}{r}
\overline{X\left(e_{0} \beta \alpha \gamma \delta\right)}, \overline{X\left(e_{0} \gamma \alpha \beta \delta\right)}, \overline{X\left(e_{0} \delta \alpha \beta \gamma\right)}, \overline{X\left(e_{0} \beta \gamma \alpha \delta\right)}, \\
\overline{X\left(e_{0} \beta \delta \alpha \gamma\right)}, \overline{X\left(e_{0} \gamma \delta \alpha \beta\right)}, \overline{X\left(e_{0} \beta \gamma \delta \alpha\right)}
\end{array}
$$

(iii) If $I=\{\beta\}$, then $M_{\text {nil }}$ has 4 irreducible components:

$$
\overline{X\left(e_{0} \gamma \alpha \beta \delta\right)}, \overline{X\left(e_{0} \delta \alpha \beta \gamma\right)}, \overline{X\left(e_{0} \gamma \delta \alpha \beta\right)}, \overline{X\left(e_{0} \alpha \beta \gamma \delta\right)}
$$

(iv) If $I=\{\alpha, \beta\}$, then $M_{\text {nil }}$ has 3 irreducible components:

$$
\overline{X\left(e_{0} \gamma \alpha \beta \delta\right)}, \overline{X\left(e_{0} \delta \alpha \beta \gamma\right)}, \overline{X\left(e_{0} \gamma \delta \alpha \beta\right)}
$$

(v) If $I=\{\beta, \gamma\}$, then $M_{\text {nil }}$ has 2 irreducible components:

$$
\overline{X\left(e_{0} \delta \alpha \beta \gamma\right)}, \overline{X\left(e_{0} \alpha \beta \gamma \delta\right)}
$$


(vi) If $I=\{\alpha, \beta, \gamma\}$, then $M_{\text {nil }}=\overline{X\left(e_{0} \delta \alpha \beta \gamma\right)}$ is irreducible.

(vii) If $I=\{\beta, \gamma, \delta\}$, then $M_{\text {nil }}=\overline{X\left(e_{0} \alpha \beta \gamma \delta\right)}$ is irreducible.

Example 6.5 Let $\mathrm{M}$ be a $\mathcal{J}$-coirreducible monoid of type $\emptyset$. Such a monoid is called a dual canonical monoid. Let $\Lambda_{\max }=\left\{e_{0}\right\}$. Then the irreducible components of $M_{\text {nil }}$ are $\overline{X\left(e_{\alpha} c\right)}$, where $c$ is a Coxeter element of $W$.

Finally we give necessary and sufficient conditions for $M_{\text {nil }}$ to be irreducible when $M$ is a semisimple monoid,

Theorem 6.6 Let $M$ be a semisimple monoid. Then $M_{\text {nil }}$ is irreducible if and only if $M$ is a $\mathcal{J}$-coirreducible monoid of maximal type.

Proof By Theorems 4.3 and 4.4, we may assume that $M$ is $\mathcal{J}$-coirreducible of type $I$. Let $\Lambda_{\max }=\left\{e_{0}\right\}$. Let $S_{1}, \ldots, S_{t}$ be the irreducible components of $S, I_{j}=I \cap S_{j}$, $j=1, \ldots, t$. Assume first that $M$ is of maximal type and $I_{j}=S-\left\{\alpha_{j}\right\}, j=1, \ldots, t$. Then by Lemma 3.2, $y=c_{S_{1}}\left(\alpha_{1}\right) \cdots c_{S_{t}}\left(\alpha_{t}\right)$ is the unique Coxeter element of $W$ in $D_{I}^{-1}$. By Theorem 6.2, $M_{n i l}=\overline{X\left(e_{0} y\right)}$ is irreducible.

Assume conversely that $M$ is not of maximal type. Let $\alpha_{i} \in S_{i}-I_{i}, i=1, \ldots, t$. Then for some $j$, there exists $\alpha_{j}^{\prime} \in S_{j}-I_{j}, \alpha_{j} \neq \alpha_{j}^{\prime}$. By Lemma 3.2, $z_{i}=c_{S_{i}}\left(\alpha_{i}\right)$, $i=1, \ldots, t$ and $z_{j}^{\prime}=c_{S_{j}}\left(\alpha_{j}^{\prime}\right)$ are in $D_{I}^{-1}$. Moreover

$$
\alpha_{j} z_{j}<z_{j}, \alpha_{j} z_{j}^{\prime}>z_{j}^{\prime}
$$

Hence $z_{j} \neq z_{j}^{\prime}$. Thus

$$
z=z_{1} \cdots z_{j} \cdots z_{t}, z^{\prime}=z_{1} \cdots z_{j}^{\prime} \cdots z_{t}
$$

are Coxeter elements of $W$ in $D_{I}^{-1}, z \neq z^{\prime}$. By Theorem 6.2, $M_{\text {nil }}$ has at least two irreducible components, This completes the proof.

\section{References}

1. Augustine, M.K., Putcha, M.S.: Maps into Dynkin diagrams arising from regular monoids. J. Aust. Math. Soc. Ser. A 47, 313-321 (1989)

2. Carter, R.W.: Finite Groups of Lie Type: Conjugacy Classes and Complex Characters. Wiley, New York (1985)

3. Chevalley, C.: Sur les décompositions cellulaires des espaces $G / B$. In: Algebraic Groups and Their Generalizations. Proc. Symp. Pure Math., vol. 56, pp. 1-23. Am. Math. Soc., Providence (1991)

4. Humphreys, J.E.: Reflection Groups and Coxeter Groups. Cambridge Univ. Press, Cambridge (1990)

5. Pennell, E.A., Putcha, M.S., Renner, L.E.: Analogue of the Bruhat-Chevalley order for reductive monoids. J. Algebra 196, 339-368 (1997)

6. Putcha, M.S.: A semigroup approach to linear algebraic groups. J. Algebra 80, 164-185 (1983)

7. Putcha, M.S.: Regular linear algebraic monoids. Trans. Am. Math. Soc. 290, 615-626 (1985)

8. Putcha, M.S.: Conjugacy classes in algebraic monoids. Trans. Am. Math. Soc. 303, 529-540 (1987)

9. Putcha, M.S.: Linear Algebraic Monoids. London Math. Soc. Lecture Note Series, vol. 133. Cambridge Univ. Press, Cambridge (1988)

10. Putcha, M.S.: Conjugacy classes and nilpotent variety of a reductive monoid. Can. J. Math. 50, 829844 (1998) 
11. Putcha, M.S.: Shellability in reductive monoids. Trans. Am. Math. Soc. 354, 413-426 (2002)

12. Putcha, M.S.: Bruhat-Chevalley order in reductive monoids. J. Algebr. Comb. 20, 33-53 (2004)

13. Putcha, M.S.: Conjugacy decomposition of reductive monoids. Math. Z. 250, 841-853 (2005)

14. Putcha, M.S., Renner, L.E.: The system of idempotents and the lattice of $\mathcal{J}$-classes of reductive algebraic monoids. J. Algebra 116, 385-399 (1988)

15. Renner, L.E.: Analogue of the Bruhat decomposition for reductive algebraic monoids. J. Algebra 101, 303-338 (1986)

16. Renner, L.E.: Linear Algebraic Monoids. Encyclopedia of Mathematical Sciences, vol. 134. Springer, New York (2005)

17. Solomon, L.: An introduction to reductive monoids. In: Fountain, J. (ed.) Semigroups, Formal Languages and Groups, pp. 353-367. Kluwer, Dordrecht (1995) 